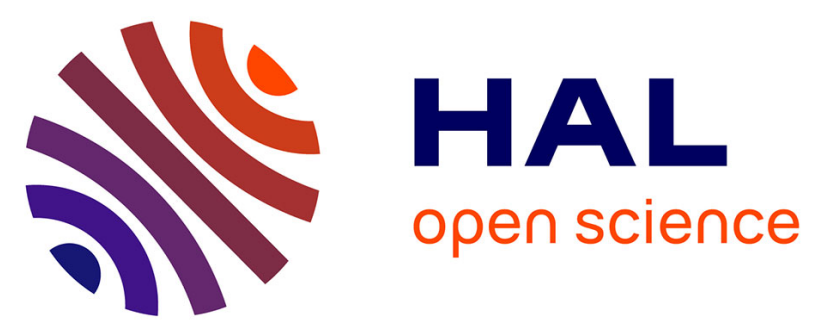

\title{
Analysis of a simple model for post-impact dynamics active compliance in humanoids falls with nonlinear optimization
}

\author{
Vincent Samy, Karim Bouyarmane, Abderrahmane Kheddar
}

\section{To cite this version:}

Vincent Samy, Karim Bouyarmane, Abderrahmane Kheddar. Analysis of a simple model for postimpact dynamics active compliance in humanoids falls with nonlinear optimization. IEEE International Conference on Simulation, Modeling, and Programming for Autonomous Robots (SIMPAR 2018), May 2018, Brisbane, Australia. pp.62-67, 10.1109/SIMPAR.2018.8376272 . lirmm-03131379

\section{HAL Id: lirmm-03131379 \\ https://hal-lirmm.ccsd.cnrs.fr/lirmm-03131379}

Submitted on 4 Feb 2021

HAL is a multi-disciplinary open access archive for the deposit and dissemination of scientific research documents, whether they are published or not. The documents may come from teaching and research institutions in France or abroad, or from public or private research centers.
L'archive ouverte pluridisciplinaire HAL, est destinée au dépôt et à la diffusion de documents scientifiques de niveau recherche, publiés ou non, émanant des établissements d'enseignement et de recherche français ou étrangers, des laboratoires publics ou privés. 


\title{
Analysis of a simple model for post-impact dynamics active compliance in humanoids falls with nonlinear optimization
}

\author{
Vincent Samy ${ }^{1}$, Karim Bouyarmane ${ }^{2}$ and Abderrahmane Kheddar ${ }^{1,3}$
}

\begin{abstract}
We analyse a mass-spring-damper model as an active compliance steering controller to adaptively comply with post-impact dynamics in humanoid falls. We use it as a one degree of freedom virtual link that can be attached between a point at impact and a given limb point (e.g. torso or waist of the humanoid). By mapping position and torque limits of the robot joints into corresponding position and force limits in the virtual link, we formulate a nonlinear optimization problem to find its admissible stiffness and damping that prevents violating the constraints before reaching a steady state rest. The nonlinear constraints are analytically derived using symbolic computation and then numerically solved with off-the-shelf nonlinear optimization solver. The virtual model trajectories are then mapped back on the full body of the humanoid robot and illustrated on the HRP-4 robot in simulation.
\end{abstract}

\section{INTRODUCTION}

Simple models, such as the center-of-mass (CoM) and multi-dimensional mass-spring-dampers, proved to be extremely efficient in capturing the dominant behavior of complex dynamical systems, among which humanoid robots. For example, the CoM and related centroidal dynamics are used to design various strategies for dynamic walking, e.g. [1], [2]. The linear inverted pendulum (LIP) model is one of the most popular in humanoid research since many years [3]. The CoM model enhanced by a spring along the massless $\mathrm{CoM} / \mathrm{Center}$ of Pressure point system (spring-loaded inverted pendulum, or SLIP) is used to generate behaviors beyond walking, e.g. jumping and running [4], [5], [6]. The CoM models were extended to generate multi-contact motions, e.g. [7], [8], [9]. Mass-spring-damper models have also been used as nominal desired impedance or admittance at the contact space [10]. There are of course many other variants of simple dynamics models and their usages in robotics.

In our previous work [11], we have addressed humanoid falling (see references in [11], [12] for a thorough state-ofthe-art approaches in humanoid falls) using locally linearized control to comply with post-impact dynamics using taskspace quadratic programming (QP) formalism [13], [14]. The idea is to reshape the humanoid posture so as to meet the impacts at contact positions that maximize compliance by adapting the actuators Proportional-Derivative (PD) gains. In [12], we showed that the actuator gain parameters can

This work is supported in parts from grants of the COMANOID EU H2020 project, of the of the Japan Society for Promotion of Science (JSPS) Grant-in-Aid for Scientific Research, Kakenhi B No. 16H02886 and of the ROMEO 2 French PSPC bpifrance project.

${ }^{1}$ CNRS-AIST Joint Robotics Laboratory, UMI3218/RL, Japan.

${ }^{2}$ Université de Lorraine, Inria, CNRS, Loria UMR 7503, Larsen team, France.

${ }^{3}$ CNRS-University of Montpellier LIRMM, Interactive Digital Human team, France.

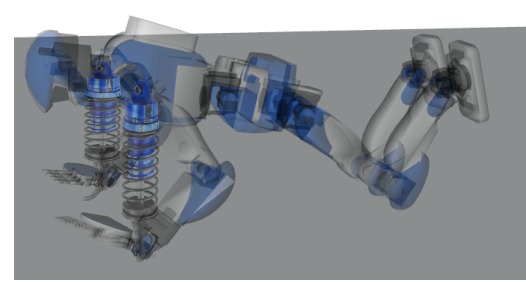

(a) Landing on arms.

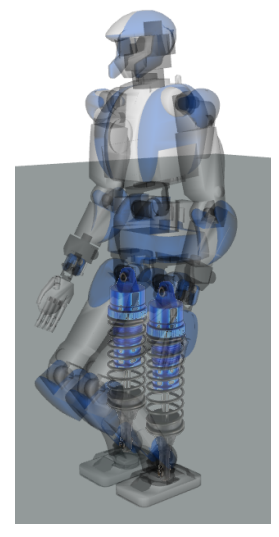

(b) Landing on legs.
Fig. 1: Shock-absorber virtual-link dynamic model representation in two different landing configurations.

also be integrated as decision variables in the QP controller. However, a problem that still needed to be solved was the computation of motor PD gains using a prediction of the system over a receding horizon (preview) to ensure that the structural limits of the robot (torque limits, joint limits) are not reached during the whole post-impact phase, until the system comes to a rest.

Inspired by what is well established in dynamic walking, we propose a mass-spring-damper model applied to the situation of humanoid falls, to be used in a two-stage QP control approach. The idea of the virtual-link dynamic model is to emulate a shock absorber on the impacting limbs of the robot (see Fig. 1). This paper is not about impact regulation or impact handling that should include robust hardware design instead. Our model shall be seen as a planning or preview control strategy to handle post-impact dynamics by actively complying assuming a system to detect and handle impacts readily available or implemented, e.g. [15].

To this end, we present a detailed analysis of the parameter tuning of a one degree-of-freedom (1 dof) massspring-damper system to serve as a model-preview adaptive controller for post-impact trajectory generation of humanoid whole-body motion after the impact occurred. We analytically derive the constraints on the stiffness and damping decision parameters of this model and formulate a nonlinear optimization problem to solve. Once the latter parameters are obtained, the corresponding trajectory is tracked by the robot in the post-impact phase using a whole-body QP controller. 


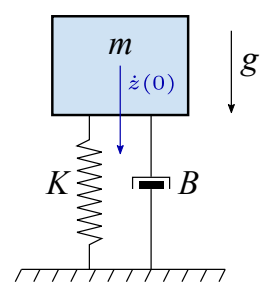

Fig. 2: Mass-spring-damper system.

\section{AnAlytical Resolution}

Let us consider the system in Fig. 2. The analytical solution for the motion of this system comes from the standard resolution of the well-studied second order linear differential equation:

$$
m \ddot{z}(t)-K\left(z^{\text {ref }}-z(t)\right)-B\left(\dot{z}^{\text {ref }}-\dot{z}(t)\right)+m g=0,
$$

where $m$ is the mass of the system, $z(t)$ is the position of the mass at time $t, g$ the gravity and $K, B$ the gains that we want to compute to fullfill the constraints that are deduced from torque and joint angle limits of the full system. This equation can also be interpreted as a PD controller of a linear motor supporting a mass $m$ under the gravity $g$. In such case, $z^{\text {ref }}$ is the reference position which is set to the position just before impact $q^{-}(0)$ and $\dot{z}^{\text {ref }}$ is always 0 .

Solving for $z(t)$ leads to known three cases: (i) an underdamped solution if $B^{2}<4 m K$ and the system oscillates at its natural frequency; (ii) critically damped solution when $B^{2}=4 m K$, it is the fastest way to reach the $z^{\text {ref }}$ position without oscillating; and (iii) the over-damped solution when $B^{2}>4 m K$, where $z^{\text {ref }}$ is reached over a longer time.

Given a solution of Eq. (1) that depends on the parameters $K$ and $B$, our objective is to find admissible values of these parameters such that:

- joint limit constraint: a position limit constraint is satisfied on $z(t)$ (e.g. preventing the mass from colliding with the ground after the impact, or equivalently reaching a maximum compression limit);

- torque limit constraint: a force limit constraint is satisfied on $K\left(z^{\text {ref }}-z(t)\right)+B\left(\dot{z}^{\text {ref }}-\dot{z}(t)\right)$ (the post-impact

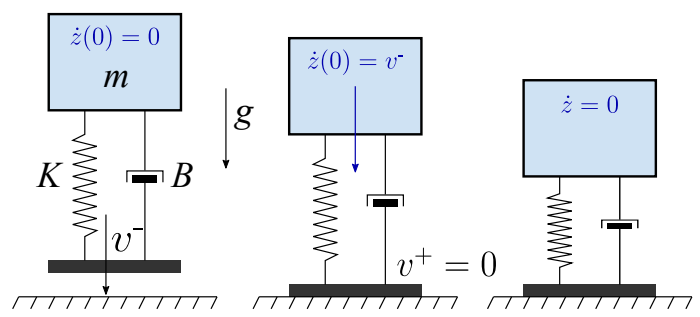

Fig. 3: Landing phases and post-impact of the virtual link. Left figure: the system just before impact at time $t=0^{-}$, the velocity of the base is $v^{-}$and the joint velocity is 0 (velocity of the mass relative to the base). Middle figure: the system just after impact at $t=0^{+}$(assumed handled by hardware at $t=0$ ). Right figure: the system at the time of maximum compression amplitude $t=t^{\mathrm{ma}}$. force generated by the spring recoil should be kept below a safety threshold when possible);

- the system converges to zero velocity.

The above constraints capture in the 1dof virtual-link the full-body constraints on both joints angle and torques limits.

We choose to search for over-damped solutions in order (i) to avoid oscillations of the virtual link that would otherwise be mapped to the whole-body, and (ii) to relax critical convergence to $z^{\text {ref }}$, as long as we reach a rest steady state over time. Hence, we solve Eq. (1) with $B^{2}>4 m K$. We will see later that this is not a limiting assumption in terms of finding a solution whenever the solution set is nonempty.

Note that we do not consider an explicit velocity constraint in the formulation, as during the entire post-impact phase, the system is only decelerating (slowing down) due to the dissipation of energy through the damping. The constraint would be effective if written on the initial velocity, but the latter is an input to the problem over which we have no direct control. Hence, the post-impact initial velocity might or might not cross the velocity limit if it was to be set.

Solving the differential equation Eq. (1) for $z$ as a function of $t$, with $K$ and $B$ as parameters, leads to:

$$
\begin{gathered}
z(t, K, B)=\frac{\mathrm{e}^{-\frac{1}{2 m}(B-\sqrt{\gamma}) t}(B g+2 K \dot{z}(0)+\sqrt{\gamma} g) m}{2 \sqrt{\gamma} K} \\
-\frac{\mathrm{e}^{-\frac{1}{2 m}(B+\sqrt{\gamma}) t}(B g-2 K \dot{z}(0)-\sqrt{\gamma} g) m}{2 \sqrt{\gamma} K}+\frac{K z(0)-m g}{K},
\end{gathered}
$$

with $\gamma=B^{2}-4 K m$.

In the following, for the sake of readability and in order to reduce the size of the symbolic expressions, but without loss of generality, we set example numerical values to the initial conditions and to the mass of the system (all the developments can be kept with their symbolic expressions):

$$
m=1, g=9.81, z(0)=0, \dot{z}(0)=-5 \text {. }
$$

The derivations and reasoning to follow are still valid for any other numerical values corresponding to the specific robot and falling conditions at hand. Initializing the velocity to a value different from zero simulates a falling system state just after an impact. Suppose the system is impacting with a net velocity $v^{-}=-5 \mathrm{~ms}^{-1}$ (external floating-base velocity) and $\dot{z}^{-}(0)=0 \mathrm{~ms}^{-1}$ (internal joint velocity) and that the impact is inelastic (no bounce), the remaining velocity in the joint post-impact is then $\dot{z}^{+}(0)=-5 \mathrm{~ms}^{-1}$, see Fig. 3 .

With the numerical values (3), $z(t, K, B)$ in (2) becomes:

$$
\begin{aligned}
z(t, K, B)= & \frac{\mathrm{e}^{-\frac{1}{2}(B-\sqrt{\gamma}) t}(-10 K+9.81(B+\sqrt{\gamma})}{2 \sqrt{\gamma} K}- \\
& \frac{\mathrm{e}^{-\frac{1}{2}(B+\sqrt{\gamma}) t}(10 K+9.81(B-\sqrt{\gamma})}{2 \sqrt{\gamma} K}-\frac{9.81}{K},
\end{aligned}
$$

with $\gamma=B^{2}-4 K$ (we recall that $\gamma>0$ ).

A 3D time-evolution representation of $z$ as function of $K$ and $B$ can be represented, this is illustrated in Fig. 4. 


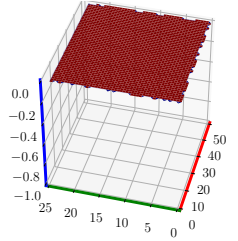

(a) $t=0 \mathrm{~ms}$

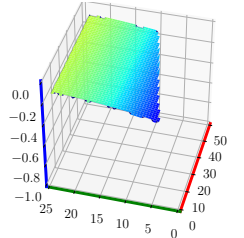

(b) $t=50 \mathrm{~ms}$

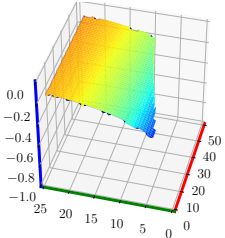

(c) $t=100 \mathrm{~ms}$

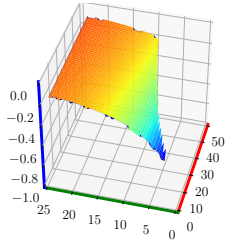

(d) $t=150 \mathrm{~ms}$

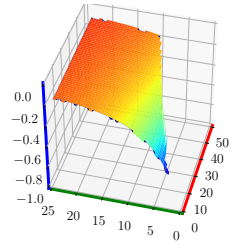

(e) $t=200 \mathrm{~ms}$
Fig. 4: Evolution of position $z$ in $\mathrm{m}$ (blue axis), for a given stiffness $K \in[0 \ldots 50]$ in N.m $\mathrm{m}^{-1}$ (red axis), and damping $B \in$ $[0 \ldots 25]$ in N.s.m ${ }^{-1}$ (green axis).

Taking the time-derivative of $z(t, K, B)$ gives us the joint velocity:

$$
\begin{aligned}
& \dot{z}(t, K, B)= \\
& \quad \frac{(-B+\sqrt{\gamma}) \mathrm{e}^{-\frac{1}{2}(B-\sqrt{\gamma}) t}(-10 K+9.81(B+\sqrt{\gamma}))}{4 \sqrt{\gamma} K} \\
& -\frac{(-B-\sqrt{\gamma}) \mathrm{e}^{-\frac{1}{2}(B+\sqrt{\gamma}) t}(10 K+9.81(B-\sqrt{\gamma}))}{4 \sqrt{\gamma} K} .
\end{aligned}
$$

The 3D time-evolution of $\dot{z}$ as a function of $K$ and $B$ can also be visualized (Fig. 5).

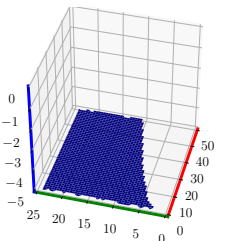

(a) $t=0 \mathrm{~ms}$

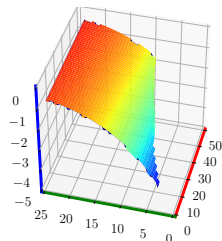

(b) $t=50 \mathrm{~ms}$

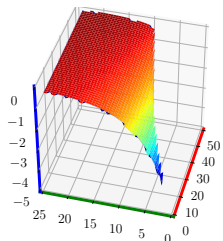

(c) $t=100 \mathrm{~ms}$

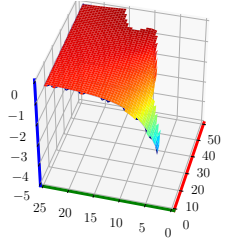

(d) $t=150 \mathrm{~ms}$

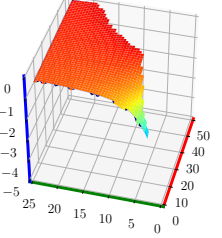

(e) $t=200 \mathrm{~ms}$
Fig. 5: Evolution of velocity $\dot{z}$ in $\mathrm{m}^{-1} \mathrm{~s}^{-1}$ (blue axis), for a given stiffness $K \in[0 \ldots 50]$ in N.m ${ }^{-1}$ (red axis), and damping $B \in[0 \ldots 25]$ in N.s.m ${ }^{-1}$ (green axis).

Lastly, from $z$ and $\dot{z}$, we can compute the joint force (spring force) with respect to time and as a function of $K$ and $B$ (Eq. (6) and Fig. 6).

$$
\begin{gathered}
f(t, K, B)=K\left(z^{\mathrm{ref}}-z(t, K, B)\right)-B \dot{z}(t, K, B)= \\
-\frac{\left(2.5 B^{2}-5 K-4.905 B+(4.905-2.5 B) \sqrt{\gamma}\right) \mathrm{e}^{-\frac{1}{2}(B-\sqrt{\gamma}) t}}{\sqrt{\gamma}} \\
-\frac{\left(-2.5 B^{2}+5 K+4.905 B+(4.905-2.5 B) \sqrt{\gamma}\right) \mathrm{e}^{-\frac{1}{2}(B+\sqrt{\gamma}) t}}{\sqrt{\gamma}}+9.81 .
\end{gathered}
$$

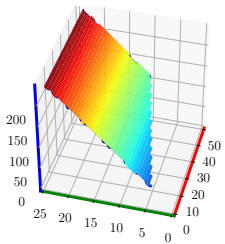

(a) $t=0 \mathrm{~ms}$

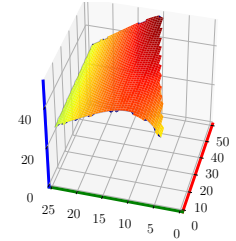

(b) $t=50 \mathrm{~ms}$

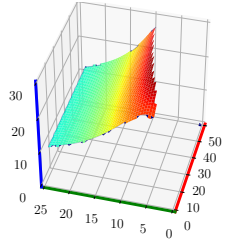

(c) $t=100 \mathrm{~ms}$

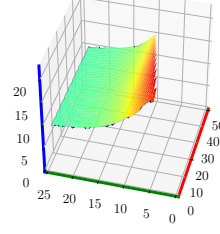

(d) $t=150 \mathrm{~ms}$

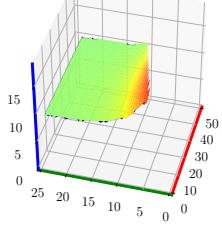

(e) $t=200 \mathrm{~ms}$
Fig. 6: Evolution of force $f$ in $\mathrm{N}$ (blue axis), for a given stiffness $K \in[0 \ldots 50]$ in N.m-1 (red axis), and damping $B \in$ $[0 \ldots 25]$ in N.s.m ${ }^{-1}$ (green axis).

The joint limit and force limit constraints can be respectively written from the expressions (4) and (6) as

$$
\begin{array}{ll}
\forall t>0 & \underline{z} \leq z(t, K, B) \leq \bar{z}, \\
\forall t>0 & \underline{f} \leq f(t, K, B) \leq \bar{f},
\end{array}
$$

where $\underline{z}, \bar{z}, f, \bar{f}$ are lower and upper bounds of the virtual link position and force deduced from joint and torque limits constraints of the robot impacting limb (see Section III).

The projection of the joint and the torque constraints on the $K-B$ plane through time is visualized in Fig. 7. The joint limit visualization is obtained by intersecting the $3 \mathrm{D}$ time-evolution plot of $z(t, K, B)$ with the plane $z=\underline{z}$, while the force limit constraint visualization is obtained by intersecting the 3D time-evolution plot of $f(t, K, B)$ with the plane at $f=\bar{f}$ (the reason why only one side of these two constraints is relevant is explained below). By analyzing these visualizations, we can substitute the time-continuous constraints (7) and (8) with fixed time constraints.

First, we can define the maximum amplitude time $t^{\mathrm{ma}}$ as the time at which the velocity reaches 0 . Solving for Eq. (5) $=0$ with $K$ and $B$ as parameters using a symbolic computation software (e.g. Maple), we get:

$t^{\mathrm{ma}}=\frac{\ln \left(-\frac{4905 B-2500 K-9623.61}{1250 B^{2}-1250 B \sqrt{\gamma}-2500 K-4905 B+4905 \sqrt{\gamma}+9623.61}\right)}{\sqrt{\gamma}}$. 


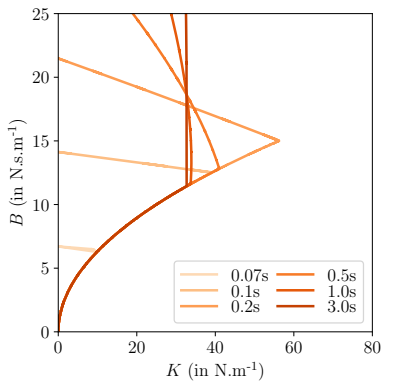

(a) Joint limit projection.

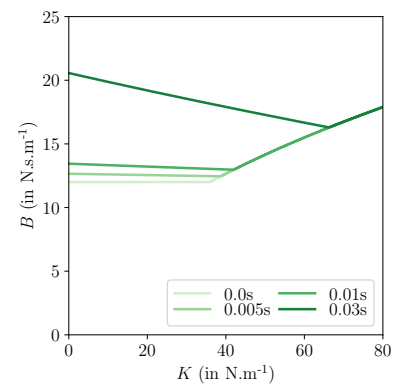

(b) Force limit projection.
Fig. 7: Projection of the joint limit and the force limit on the $K-B$ plane for different time with $\underline{z}=-0.3 \mathrm{~m}$ and $\bar{f}=60 \mathrm{~N}$.

This equation is stiffness-dependant and damping-dependant. The joint limit constraint can then be captured by:

$$
\underline{z} \leq z\left(t=t^{\mathrm{ma}}, K, B\right) \leq \bar{z} .
$$

Secondly, we observe that the force limit can be reached in three main ways: i) high damping force when having high velocity; ii) high stiffness force when having high position error; iii) a mix of the two. In this study, we are interested in post-impact dynamics involving a relatively short compression range between initial position and the limit, and high post-impact velocity resulting from the fall. In these conditions, the force maximum value is more likely to come from the damping of the velocity and not from the stiffness related to the distance. And since we only decelerate the system, we consider that the maximum force is reached at $t=0^{+}$(after impulse). The torque limit constraint (here a force limit constraint) thus writes:

$$
\underline{f} \leq f\left(t=0^{+}, K, B\right) \leq \bar{f} .
$$

Of course, it is necessary to confirm this latter assumption once the $t \mapsto f(t)$ trajectory is derived with the computed values of $K$ and $B$. This is the case in Fig. 7b.

As only the over-damped behavior is accounted, a third constraint, the over-damped system constraint, is added to the above joint limit and torque limit constraints.

$$
4 K-B^{2}<0 .
$$

The problem we have formulated up to this point thus reduces to the following system of inequalities:

$$
\begin{aligned}
& \text { find } K, B \\
& \text { such that }\left\{\begin{array}{l}
\underline{z} \leq z\left(t=t^{\mathrm{ma}}, K, B\right) \leq \bar{z} \\
\frac{f}{4} \leq f\left(t=0^{+}, K, B\right) \leq \bar{f}
\end{array}\right.
\end{aligned}
$$

This is a system of nonlinear inequalities, as the constraints (10)-(12) are nonlinear inequalities in $K$ and $B$, of which we have derived the analytical expressions throughout this section using symbolic computation tools.

To solve our system of nonlinear inequalities, we formulate it as the system of constraints of a nonlinear constrained optimization problem in $K$ and $B$, with an arbitrary convex cost function, e.g. choose the gains to be close to the nominal PD gains $K_{n}$ and $B_{n}$ used in whole-body control, by minimizing $\left(K-K_{n}\right)^{2}+\left(B-B_{n}\right)^{2}$; or since we want the most passive joint possible, we can alternatively minimize:

$$
\begin{aligned}
& \min _{K, B} K^{2}+B^{2} \\
& \text { such that }\left\{\begin{array}{l}
\underline{z} \leq z\left(t=t^{\mathrm{ma}}, K, B\right) \leq \bar{z} \\
\frac{f}{4} \leq f\left(t=0^{+}, K, B\right) \leq \bar{f} \\
4 K-B^{2}<0
\end{array}\right.
\end{aligned}
$$

that finds the minimum norm solution for $(K, B)$ satisfying the system of nonlinear inequality constraints.

We use the Matlab software to compute the solution of this optimization problem. The result is shown in Fig. 8. In this example, the impulse happens at the time $t_{0}=0 \mathrm{such}$ that at $t_{0}^{-}$the velocity is zero and at $t_{0}^{+}$the velocity is $-5 \mathrm{~m} \cdot \mathrm{s}^{-1}$ and $z(0)=0, \underline{z}=-0.5, \bar{f}=60$.

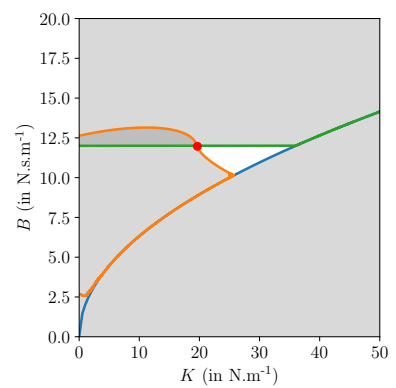

(a) Solution region $(\underline{z}=-0.5)$

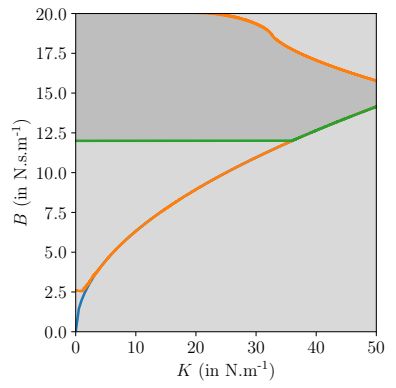

(b) No solution $(\underline{z}=-0.3)$
Fig. 8: Computation of the solution region depending on the problem constraints in the $K-B$ domain. The blue curve represents Eq. (12), the green curve Eq. (11) and the orange curve Eq. (10). The set of admissible solutions is the white region whereas the red dot is the particular solution, of the the nonlinear optimization problem.

When the joint limit is not too close to the current position (Fig. 8a), we are able to find a set of solutions that satisfy all the constraints (Fig. 9). However, if the joint limit is set to 0.3 instead of 0.5 (much closer to the current position) as in Fig. 8b, no solution can be found, as the solution set is empty. The latter situation can also occur in falling conditions from which it is impossible for the robot to safely comply (e.g. dropping from a high altitude, impacting with very high velocity).

\section{RESULTS}

The accompanying video shows simulations that were realized based on our approach; first with low-dimensional toy systems (matching the studied model) and on a full humanoid robot HRP-4.

The Fig. 10a is a trivial application of our method on a 1-dof system: the virtual link corresponds to the full model. The Fig. 10b shows a 2-dof system with two prismatic joints. The maximum force $\bar{f}$ is the sum (and not the least) of the maximum forces of the two linear actuators, and the 


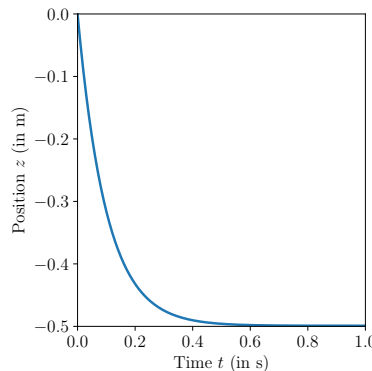

(a) Evolution of $z(t)$.

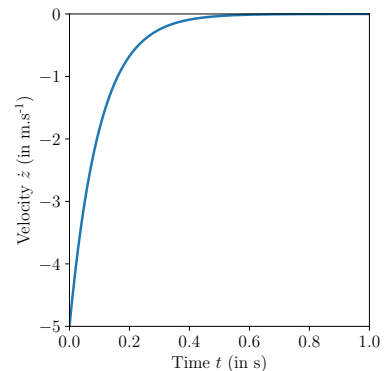

(b) Evolution of $\dot{z}(t)$.

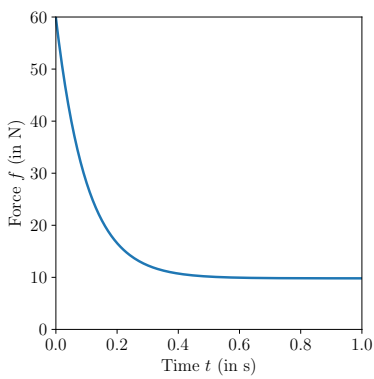

(c) Evolution of $f(t)$.

Fig. 9: Evolution of the different joint parameters through time. The system reaches the joint limit without crossing it and after around $0.5 \mathrm{~s}$ it is completely stopped.

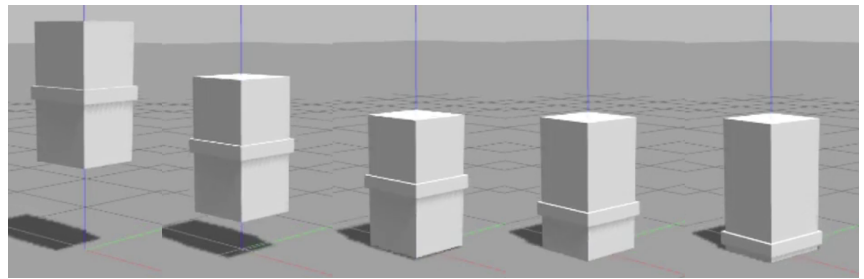

(a) 1-dof system simulation.

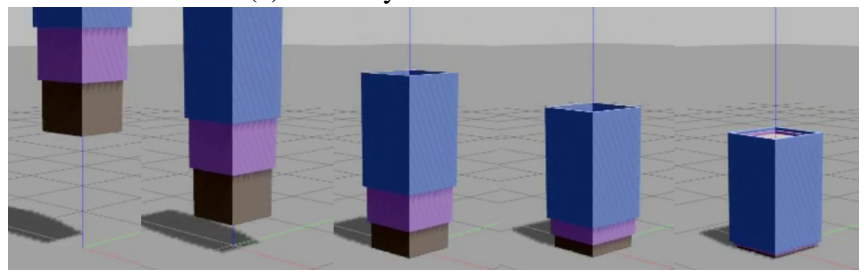

(b) 2-dof system simulation.

Fig. 10: Low-dimensional systems simulations.

minimum position $\underline{z}$ is obtained when the two joints are put at their position limits. In both examples, we obtain a perfect realization of the desired behavior in simulation, after both systems reach the ground with an impact velocity of $v^{-}=$ $-5 \mathrm{~ms}^{-1}$.

The Figs. 11 and 12 show falling motions of the humanoid HRP-4, controlled based on the virtual-link model illustrated in Fig. 1. The virtual link model analysis is used to generate trajectories for the limb extremity attached to torso (arms) or to the waist (legs), and these trajectories are tracked in the post impact phase with a whole-body QP controller.

In these two cases, the limit position $\underline{z}$ of the virtual link

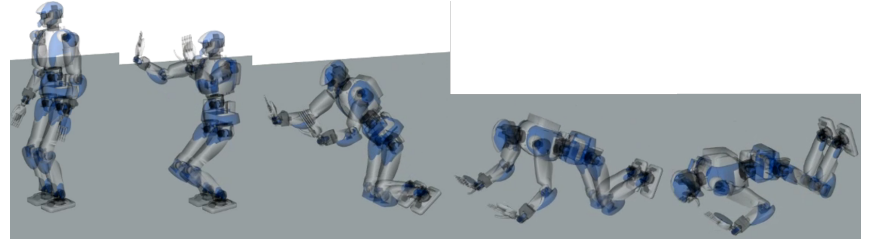

Fig. 11: Front fall screenshots from the accompanying video.

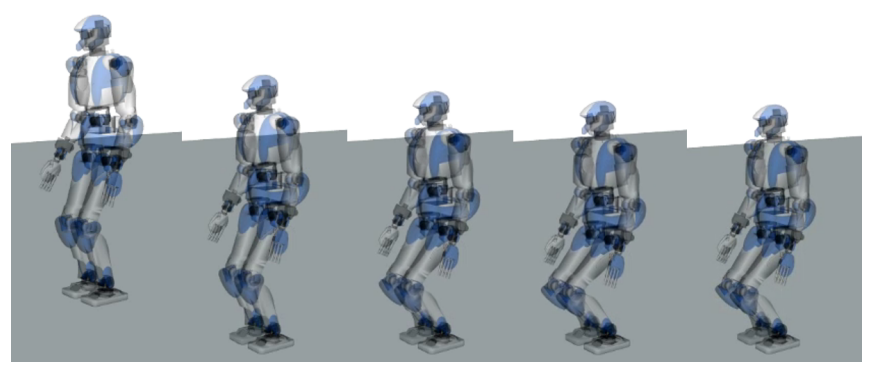

Fig. 12: Leg fall screenshots from the accompanying video.

is obtained from forward kinematics of the limb of interest when all the joints are put in their limits:

$$
\underline{z}=\mathrm{FK}^{\text {limb }}\left(q_{\text {limit }}^{\text {limb }}\right),
$$

where $\mathrm{FK}^{\mathrm{limb}}$ denotes the forward kinematics function of the limb and $q_{\text {limit }}^{\text {limb }}$ the configuration of the limb at its limits (here $\operatorname{limb} \in\{\mathrm{arm}, \operatorname{leg}\})$. However, it is only an approximation, since $\mathrm{FK}^{\mathrm{limb}}$ is generally a nonlinear mapping. This example shows that obtaining the exact bounds on the virtual-link range of motion (i.e. the compression limit) from the robot joint limits and the kinematics is not trivial (see Section IV). For the force bound of the virtual link, it can be obtained in first approximation from the pseudo inverse of the Jacobian transpose applied on the torque limits of the limb, or exactly by using the force polytope analysis detailed in [16], which accounts for both torque limits in the limb and friction limits at the contact. As for the effective mass of the virtual link in

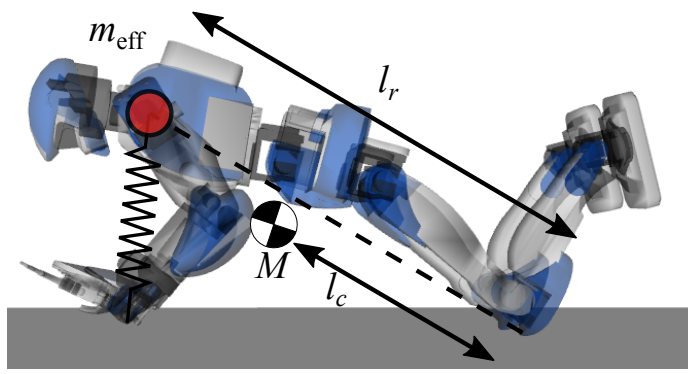

Fig. 13: Effective mass computation.

case of landing on the arms, we use the reasoning illustrated in Fig. 13. Modeling the part of the robot between the knee and the arm as an inverted pendulum rod, the effective mass can be defined as the point mass localized at the extremity of the rod that has the same moment around the CoP of the rod as the total gravity force applied at the CoM, i.e.

$$
M g l_{c} \cos (\theta)=m_{\mathrm{eff}} g l_{r} \cos (\theta),
$$


where $M$ is the mass of the rod (mass of the robot between the knee and the arm), $l_{c}$ the distance of the CoM of the rod from its CoP at the knee, $l_{r}$ the total length of the rod (distance between the effective mass and the $\mathrm{CoP}$ ), and $\theta$ the angle between the rod and and ground. Therefore, we have:

$$
m_{\mathrm{eff}}=M \frac{l_{c}}{l_{r}} .
$$

The impact velocities for the arm landing and leg landing falls were respectively measured at $v^{-}=-3 \mathrm{~ms}^{-1}$ and $v^{-}=-1.47 \mathrm{~ms}^{-1}$. In Fig. 11, the particular posture that the robot adopts just before it starts falling is generated by applying the method presented in our previous work on nonsingular falls and optimal falling postures [11], [12].

\section{CONCLUSiOn AND Discussion}

We presented a study on a one degree of freedom massspring-damping model, called virtual link, to capture the dynamics of active post-impact compliance when a humanoid robot falls and collides with the environment.

The analysis of the system allowed us to find the stiffness and damping parameters $(K, B)$ of the virtual link model that satisfy, when possible, the structural robot limits that are the joint limits and torque limits mapped onto a position limit and force limit on the virtual link. Our approach results in trajectories that are subsequently tracked by a wholebody QP controller, simulating the behavior needed by the humanoid to realize active compliance to the post-impact dynamics, using an admittance task to map obtained forces.

As discussed in Section III, a general exact mapping from the joint limits of a humanoid robot to the bounds on the range of motion of the corresponding virtual link can be difficult to obtain. A good approximation and practical method consisted in using forward kinematics and reachable space computation of the point corresponding to the attachment of the mass considering the contact constraint. This is the method we used in our examples. An alternative method would be to represent the kinematic tree structure of the humanoid as a deformable structure (spring-loaded joints) with repulsive potential fields at the joint limits, and use computer graphics animation techniques (such as a virtual force pulling the virtual link's attachment point along the gravity until equilibrium is reached) to compute the desired bounds iteratively. This can be obtained with very fast simple computations, which we investigate in future work.

Our analysis highlighted situations in which no solution exists (when the solution set on $(K, B)$ is empty), due to extreme falling conditions for example (high impact velocity) or very limiting bounds. In these situations, the proposed approach is unsuccessful and the model appears to be no longer valid to propose a practical damage-reduction solution. Future work will investigate what alternative models can be used to deal with these extreme situations.

Moreover, other limiting situations in which a constant $(K, B)$ solution does not fit in the constraint region throughout the time can also occur. It can be overcome in future work by seeking for time-varying $(K(t), B(t))$. We can optimize for those with trajectory optimization techniques using basisfunctions parameterization (e.g. splines).

The approach in this work was specifically designed for position-controlled robots, and applied in simulation by tracking the desired trajectories by a whole-body controller with high motor PD gains. We plan in the future to combine this work with our previous work on low-level motor PD gain adaptation [12] to realize the tracking of the trajectories with lower adaptive motor PD gains.

Finally, we plan to extend the analysis proposed here to directly tune the low-level motor PD gains of the joints by using a multi-dof system modeling the compliance with a torsional spring-damper behavior at each joint.

\section{REFERENCES}

[1] D. E. Orin, A. Goswami, and S.-H. Lee, "Centroidal dynamics of a humanoid robot," Autonomous Robots, vol. 35, no. 2, pp. 161-176, Oct 2013.

[2] P.-B. Wieber, S. Kuindersma, and R. Tedrake, "Modeling and control of legged robots," in Handbook of Robotics, 2nd ed., B. Siciliano and O. Khatib, Eds. Springer, 2015, ch. 48.

[3] S. Kajita, F. Kanehiro, K. Kaneko, K. Fujiwara, K. Harada, K. Yokoi, and $\mathrm{H}$. Hirukawa, "Biped walking pattern generation by using preview control of zero-moment point," in IEEE International Conference on Robotics and Automation, vol. 2, 2003, pp. 1620-1626.

[4] H. Geyer, A. Seyfarth, and R. Blickhan, "Compliant leg behaviour explains basic dynamics of walking and running," Proceedings of the Royal Society of London B: Biological Sciences, vol. 273, no. 1603, pp. 2861-2867, 2006.

[5] I. Mordatch, M. de Lasa, and A. Hertzmann, "Robust physics-based locomotion using low-dimensional planning," ACM Transactions on Graphics (SIGGRAPH), vol. 29, no. 3, 2010.

[6] P. M. Wensing and D. E. Orin, "3D-SLIP steering for high-speed humanoid turns," in IEEE/RSJ International Conference on Intelligent Robots and Systems, 2014, pp. 4008-4013.

[7] I. Mordatch, E. Todorov, and Z. Popović, "Discovery of complex behaviors through contact-invariant optimization," ACM Transactions on Graphics (SIGGRAPH), vol. 31, no. 4, p. 43, 2012.

[8] H. Audren, J. Vaillant, A. Kheddar, A. Escande, K. Kaneko, and E. Yoshida, "Model preview control in multi-contact motionapplication to a humanoid robot," in IEEE/RSJ International Conference on Intelligent Robots and Systems, Sept 2014, pp. 4030-4035.

[9] H. Audren and A. Kheddar, "Model-predictive control in multicontact based on stability polyhedrons," in IEEE-RAS International Conference on Humanoids, Birmingham, UK, 15-17 November 2017, pp. 631-636.

[10] N. Hogan, "Impedance control: An approach to manipulation: Part I-theory," ASME Journal of Dynamic Systems, Measurement, and Control, vol. 107, no. 1, pp. 1-7, 31985.

[11] V. Samy and A. Kheddar, "Falls control using posture reshaping and active compliance," in IEEE-RAS International Conference on Humanoids, Seoul, Korea, 2015, pp. 908-913.

[12] V. Samy, K. Bouyarmane, and A. Kheddar, "QP-based adaptive-gains control to lower damage in humanoid falls," in IEEE International Conference on Robotics and Automation, Singapore, 29 May-3June 2017, pp. 4762-4767.

[13] J. Vaillant, K. Bouyarmane, and A. Kheddar, "Multi-character physical and behavioral interactions controller," IEEE Transactions on Visualization and Computer Graphics, vol. 23, no. 6, pp. 1650-1662, June 2017.

[14] K. Bouyarmane and A. Kheddar, "On weight-prioritized multi-task control of humanoid robots," IEEE Transactions on Automatic Control, 2018.

[15] M. Battaglia, L. Blanchet, A. Kheddar, S. Kajita, and K. Yokoi, "Combining haptic sensing with safe interaction," in IEEE/RSJ Int. Conf. on Intelligent Robotics and Systems, Saint Louis, MO, USA, 11-15 October 2009, pp. 231-236.

[16] V. Samy, S. Caron, K. Bouyarmane, and A. Kheddar, "Post-impact adaptive compliance for humanoid falls using predictive control of a reduced model," in IEEE-RAS International Conference on $\mathrm{Hu}$ manoids, Birmingham, UK, 15-17 November 2017, pp. 655-660. 